\title{
The Dark Energy Problem: An Inspiration for New Physics
}

\author{
Ishwaree P. Neupane \\ Department of Physics and Astronomy, University of Canterbury, \\ Private Bag 4800, 8041 Christchurch, New Zealand \\ ishwaree.neupane@canterbury.ac.nz
}

\begin{abstract}
As much as physics has advanced in the 20th century and the beginning of the current one, reaching astounding accuracy when comparing modern theories of particle physics and general relativity to experimental results, there has been a signi cant progress in observational and theoretical cosmology. Despite these progresses, we have not been able to account for what seems to be nearly $73 \%$ of the energy budget of the universe and hence its mystic name 'dark energy'. The dark energy problem provides an inspiration for seeking new laws or symmetries in nature: more precisely, a search for concise and fundamentally simple relationship between the 4D Planck mass and the present size of the universe (or the present value of the Hubble expansion parameter).
\end{abstract}

Cosmology is a young science - one which attempts to reconstruct and explain the entire history of the universe from nearly 14 billions of years ago [1]. This history must include an era of accelerated expansion, known as "inflation", which provides the most credible explanation to how causal physics in the early universe produced the large scale structures that we observe today. However, peering back so far in time is difficult and an added difficulty is that many of the theoretical pillars of physics upon which the models of inflation and gas physics at later times rest have only been proposed within the last 3 decades. That hasn't given both theoretical physicists and cosmologists much time to fully flesh out and comprehend the situation. The discovery that the expansion of the universe is accelerating is among the most significant of recent times.

The current understanding is that this is probably due to the present-day universe being dominated by a cosmological constant or other fluid-like "dark energy". Several independent observations [2], including large redshift surveys $[3,4]$, indicate that nearly $73 \%$ of the total energy density of the universe is in the form of dark energy and about $23 \%$ is in the form of non-baryonic cold dark matter particles which clump gravitationally, but which have never been directly detected. These scientific enigmas suggest we should look to new physics beyond Einstein's theory of General Relativity (GR) and the standard model of particle physics.

There is no shortage of ideas for how to construct a model which is capable to produce a Robertson-Walker-type cosmology with de Sitter-type expansion. There is already a large gamut of gravitational theories that can explain a period of accelerated expansion of the universe with certain modification of Einstein's theory of general relativity $[5,6]$. The issue of a late epoch cosmic acceleration is not about the difficulty of finding a particular model which could mimic as the Lambda-CDM cosmology, described by Einstein gravity with a cosmological constant and minimally coupled to both the luminous (baryonic) and non-luminous (cold dark) matter. Rather, the challenge is to come up with a fully consistent theory in 4 dimensions that explains the origin of cosmic acceleration, while providing insights into some other major problems in physics, including the mass hierarchy and the cosmological constant problems.

The smoothness of the cosmic microwave background (CMB) at the surface of last scattering ( 379,000 years after the bigbang) detected by NASA's WMAP satellites is paradoxical to the lumpiness of distribution of matter in the present universe. Further, an estimate of gravitational vacuum energy density using quantum gravity approaches reveals that our universe would double in size in every $10^{-43} \mathrm{sec}$. But observations seem to indicate that our universe has doubled in size in every $1010 \mathrm{yrs}$. This leads to another paradox, which is that $\Lambda$ obs $\sim 10^{-120} \Lambda_{\text {estimate }}$, or that why gravity is so weak on cosmological scales.

Theoretical physics thrives on crises and paradoxes, as in some other disciplines of science. To solve the problems, we often search for new ideas, physical principles or symmetries in nature! In this endeavor, theorists have found a way to reconcile gravity with 
quantum physics, but at the price of postulating extra dimensions beyond the familiar four dimensions of space and time. Such extra dimensions, which emerge naturally as we probe spacetime at shorter distances, can be of the Kaluza-Klein type (or compact), or our observed universe can be a cosmological "brane" embedded in a higher-dimensional space [7]. The main motivation for going beyond Einstein's theory of relativity comes from string theory - a mathematically consistent theory of quantum gravity in $9+1$ space-time dimensions. The best understanding of string theory is in terms of a particle theory via the incredible idea of holography [8], which

is apparently the most important theoretical discovery of last 20 years!

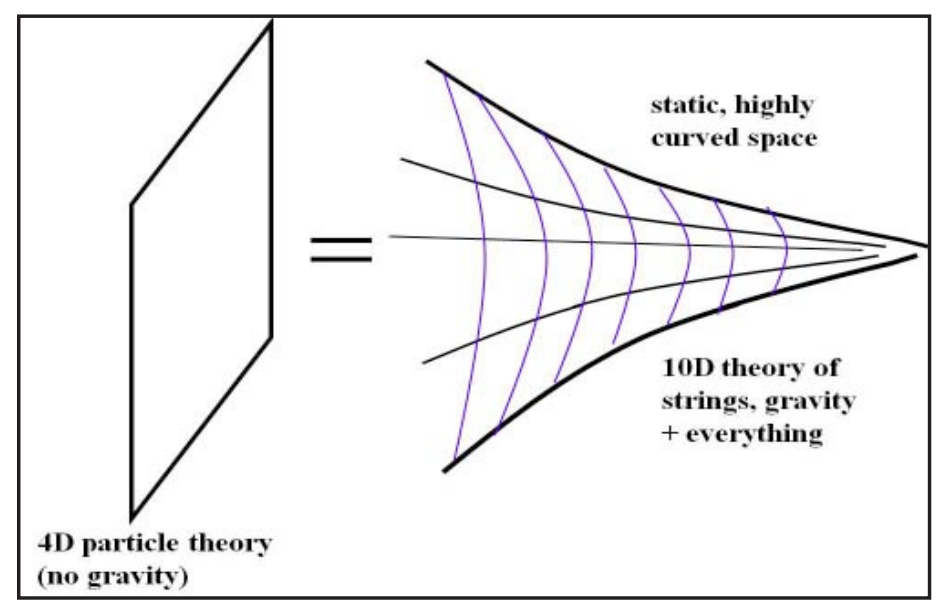

Figure 1: A holographic view of our universe

A holographic universe as simple as shown in Fig. 1 works nicely through AdS/CFT correspondence $[9,10]$ for a 4D particle theory with no gravity - that is, for a unified theory of Special Relativity and Quantum Mechanics. However, General Relativity makes spacetime dynamical and, as a result, the weight of a cosmological vacuum (or dark energy) might vary with both length and time scales. This idea brings a fundamental change to the notion of vacuum: more precisely, the dark energy that permeates empty space and accelerates the expansion of the universe must have explanations both in quantum physics and quantum gravity.

To this end, an exciting development in string cosmology is the suggestion that the warping of extra dimensions plays a key role in localizing gravity on an expanding $3+1$ spacetime, or 3-dimensional de Sitter brane, which is embedded in a higher-dimensional space. This very idea is inspired by a theoretical framework of scientific thoughts and also by fundamental theories of gravity, particles and fields. A novel feature of a brane-world-type description of the physical 3+1 spacetime is that the standard 4-dimensional gravity, not very different from Einstein's general gravity, is realized as the zero-mode solution of a 5D graviton wave equation.

As a canonical example, let us take $n=1$. If the background geometry is a warped 5D AdS space and the Hubble parameter is zero, then one encounters the simplest brane-world model proposed by Randall and Sundrum [11]. In this case we already know that there exists a massless graviton as the zero-mode solution, which reproduces the standard Newtonian gravity on the 3-brane. The Kaluza-Klein modes arising as the effect of graviton fluctuations in the $5 \mathrm{D}$ bulk AdS spacetime give rise to corrections to the Newton's force law. The brane-world models proposed in [7, 11] correspond to a static universe for which the $4 \mathrm{D}$ scale factor of the universe is constant. This setup is therefore not suitable for describing a realistic cosmological model for which space and time are dynamical and need to be treated on an equal footing. Furthermore, AdS5 is perhaps not the most preferable background geometry for a universe to pass through a de Sitter expansion at a late epoch. The main reason being that the standard 4D gravity, which may be viewed as the zero-mode solution of a 5-dimensional graviton wave equation, is not necessarily normalizable if the background spacetime is anti de Sitter.

The 5-dimensional metric that we have in mind is

$$
d s^{2}=e^{2 A}\left(\gamma_{\mu \nu} d x^{\mu} d x^{\nu}+d z^{2}\right)
$$

where $A=A(z)$, Here we look for a clas of solutions for which the 4-dimensional line elemment takes the standard FriedmannLamitre-Robertson-Walker (FLRW) form

$$
d s_{4}^{2}=\gamma_{\mu \nu} d x^{\mu} d x^{\nu}=-d t^{2}+a^{2(t)}\left[\frac{d r^{2}}{1-k r^{2}}+r^{2} d \Omega_{2}^{2}\right] \ldots
$$

where $k$ is the $3 \mathrm{D}$ curvature constant $(\mathrm{k}=0, \pm 1)$. The $5 \mathrm{D}$ gravity action takes the form

$$
S_{\text {grav }}=M_{(5)}^{3} \int d^{5} x \sqrt{-9}\left(r-2 A_{5}\right)+\int \sqrt{-\gamma}(-T)
$$

where $M_{(5)}$ is the 5D Planck mass, $T$ is the 3-brane tension and $A_{5}$ is the bulk cosmological term. In static case with $a(t) \equiv 1$, so that $d s_{4} 2=-d t^{2}+d x^{2}+d y^{2}+d z^{2}$, the zero-mode gravity solution is 
normalizable provided the 3 -brane tension satisfies $T=12 \mathrm{~m}^{3}{ }_{(5)} / \mathrm{l}$ and $A_{5}=-6 / l^{2}$, where $l$ is the radius of curvature of the $5 \mathrm{D}$ bulk spacetime. In the limit $A^{5} \rightarrow 0$, the $5 \mathrm{D}$ spacetime becomes spatially flat and gravity is not localized in this case. With $A^{5}=6 / l^{2}>0$ and $a(t) \alpha e^{H t}$, the solution for warp factor is given by

$$
\mathrm{e}^{\mathrm{A}(\mathrm{z})}=\frac{\mathrm{IH}}{\cosh \mathrm{Hz}}, \quad \mathrm{T}=\frac{6 \mathrm{M}_{(5)}^{3}}{\mathrm{I}} \sinh \mathrm{Hz} c
$$

where $z_{c}>0$. The mass reduction formula (or Gauss law) is given by

$$
M_{(5)}^{3}=\frac{2 M_{P 1}^{3}}{\pi l^{3} H^{2}}
$$

The $4 \mathrm{D}$ effective action is nothing but $\mathrm{I}=\int \mathrm{d}^{4} \sqrt{-\mathrm{g}_{4}}\left(\mathrm{R}-2 \Lambda_{4}\right.$ where $\Lambda_{4}=6 \mathrm{H}^{2}$. This result shows that, in an expanding Universe, the background spacetime can get natureally warped so as to balance the effect of a positive curavature associated with the 4D cosmological constant [12]. The zero-mode solution $\left(\mathrm{m}^{2=0)}\right.$, which is given by [13]

$\varphi_{0}(\mathrm{z})=\frac{\mathrm{b}_{0}}{(\cosh (\mathrm{Hz}))^{3 / 2}}$

is clearly normalizable, since

$$
\int_{-\infty}^{\infty}\left|\psi_{0}(z)\right|^{2} d z=\frac{\pi b_{0}^{2}}{2 H}
$$

There is one more bound state solution, i.e.

$$
\psi_{1}(z) \propto \frac{\sqrt{\cosh ^{2}(H z)-1}}{(\cosh (H z))^{3 / 2}},
$$

which corresponds to the choice $m^{2}=2 H^{2}$, where $m$ is the mass of 5D Kuluza-Klein moddes. This solution is also normalizable. However, only the zero-mode solution $\left(m^{2}=0\right)$ is localized on the de Sister brane. In the large $z H$ limit, we obtain

$$
\psi_{H z \rightarrow \infty}=c_{1} e^{i \mu z H}+c_{2} e^{-i \mu z H},
$$

Where $\mu \equiv \sqrt{\frac{m^{2}}{H^{2}}-\frac{9}{4}}$. On a cosmological scale one has $H^{-1}$ « $z$. With $\mathrm{c}_{1}=0$, all heavy modes with $\mu=0$ become oscillating plane waves and are delocalized.

Similar result exist when we supplement a 5D action with a scalar field Lagrangian, namely

$$
S_{5}=\frac{1}{2} \int d^{5} x \sqrt{-g}\left[\frac{R}{\kappa_{5}^{2}}-g^{A B} \partial_{A} \phi \partial_{B} \phi-2 U(\phi)\right],
$$

where $K_{5}{ }^{2} \equiv 1 / M_{(5)}^{3}$. In this case the solutions are given by $\phi=\phi_{0}-\kappa_{5}^{-1} \sqrt{3 \delta(1-\delta)} \sin ^{-1} \tanh (H z / \delta), \quad$ and $e^{A(z)}=e^{A_{0}}[\cosh (H z / \delta)]^{-\delta}$, where $\mathrm{A}_{0}$ and $\delta$ are some constants $(0<\delta<1)$. Furthermore, the 4-dimensional effective theory is described by the action

$$
S_{\mathrm{eff}}=\frac{M_{\mathrm{Pl}}^{2}}{2} \int d^{4} x \sqrt{-g_{4}} R_{4}-\int d^{4} x \sqrt{-g_{4}} \Lambda_{4} .
$$

The 4D Planck mass $M_{P 1}$ and the $4 \mathrm{D}$ effective cosmological constant $\Lambda_{4}$ read as

$$
M_{\mathrm{Pl}}^{2} \equiv \frac{M_{(5)}^{2} e^{3 A_{0}} \delta}{H} \frac{\sqrt{\pi} \Gamma[3 \delta / 2]}{\Gamma[(3 \delta+1) / 2]},
$$

$\Lambda_{4}=\frac{e^{3 A_{0} H}}{\kappa_{5}^{2}} \int \frac{6 \delta \cosh ^{2} \varphi-3 \delta-1}{(\cosh \varphi)^{2+3 \delta}} d \varphi$,

Where $\mu \equiv \frac{\mathrm{Hz}}{\delta}$. Note that case $\delta=1$ is special for which $\varnothing$ and hence $\mathrm{U}(\varnothing)$ are constants. In this case, as in the model without a scalar field Lagrangian, the 4D Newton's constant is finite, despite having a noncompact extra dimension. Similar results exist in a wider class of (Supergravity) models with an arbitrary number $n$ of extra dimensions $[14,15]$

Let us summarize the main points of our discussions above. The paradigm that the physical universe is a brane-like 4-dimensional hypersurface is fascinating. The important question that we attempted to address here is whether or not a canonical theory of 5D gravity can lead to a 4D description in which Einstein gravity emerges as a consistent de Sitter compactification.

Through this canonical example in $5 \mathrm{D}$, we are not arguing that the simplest braneworld-type gravitational theory may explain all major cosmological problems of recent times; shifting the emphasis from conventional explanation for dark energy to a boarder picture of D-dimensional. Einsteins theory leads to a general class of accelerating cosmologies in $3+1$ dimensions which includes Einstein's general relativity supplemented with a cosmological constant-like term, $\Lambda 4$. 
It is worth emphasizing that a canonical 5D theory considered above admits both an effective 4D Newton constant that remains finite and a normalizable graviton wave function. It is straightforward to extend the above discussions to 10 dimensions $[14,15]$, or more specifically, to string theory, which includes generalizations of both standard quantum field theory and GR. The model discussed above is sufficiently simple; it is quite possible that the unified theory of everything requires a highly symmetric, or even a supersymmetric background, such as AdS5 $\times$ S5 - a product space of a 5-dimensional Anti de Sitter space and a five-sphere. While an AdS5 background spacetime is important for studying conformal field theories - for its role in the AdS/CFT correspondence [9] - the existence of a 5-dimensional de Sitter space seems to be equally important for obtaining an effective 4D Newton constant that remains finite and a normalizable zero-mode graviton wave function. This possibly also reveals that the breaking of supersymmetry corresponds to a nucleation of the quantum world from an AdS5 to a dS5 state. It is fair to argue that in order to determine what dark energy is and why it exists requires connecting the cosmic reality of dark energy to a better fundamental understanding of microscopic quantum physics. We can see two distinct possibilities: either, we're going to find a fatal flaw in our prevailing view of the universe, especially about the existence of dark energy, or encounter interesting surprises and discoveries in cosmology in the next 1-2 decades.

\section{Acknowledgement}

This work was supported by the Marsden fund of the Royal Society of New Zealand.

\section{REFERENCES}

[1] WMAP Project Webpage [http://map.gsfc.nasa.gov].

[2] E. Komatsu, et al. 2009. [WMAP Collaboration], Astrophys. J. Suppl., 180. 330.

[3] A. G. Riess et al. 1998. [Supernova Search Team], Astron. J. 116. 1009.

[4] S. Perlmutter et al. 1999. [Supernova Cosmology Project] Astrophys. J. 517. 565.

[5] E. J. Copeland, M. Sami and S. Tsujikawa, 2006. Int. J. Mod. Phys. D 151753.

[6] B. M. Leith and I. P. Neupane, 2007. JCAP 0705019.

[7] L. Randall and R. Sundrum, 1999. Phys. Rev. Lett. 83 3370

[8] G. 't Hooft, Dimensional Reduction in Quantum Gravity, Preprint:arXiv:gr-qc/9310026.

[9] J. M. Maldacena, 1998. Adv. Theor. Math. Phys. 2231.

[10] E. Witten, 1998. Adv. Theor. Math. Phys. 2253 [arXiv:hep-th/9802150].

[11] L. Randall and R. Sundrum, 1999. Phys. Rev. Lett. 83 4690.

[12] I .P. Neupane, 2010. Int. J. Mod. Phys. D19 2281 [arXiv:1004.0254].

[13] I. P. Neupane, 2011. Phys. Rev. D83 086004 [arXiv:1011.6357].

[14] I. P. Neupane, 2010. Class. Quant. Gravity 27 045011; Phys. Lett. B683 88.

[15] I. P. Neupane, 2011. Nucl. Phys. B847 549-566 [arXiv:1011.5007]. 